\title{
AVALIAÇÃO DE GENÓTIPOS DE PESSEGUEIROS ORNAMENTAIS VISANDO AO PAISAGISMO ${ }^{1}$
}

\author{
TIAGO MADRUGA TELESCA DA SILVEIRA², MARIA DO CARMO BASSOLS RASEIRA, \\ LEONARDO MILECH ${ }^{4}$
}

RESUMO - O pessegueiro é, geralmente, cultivado por causa dos frutos que produz. Entretanto, há os que se cultivam como plantas ornamentais e, inclusive, há programas de melhoramento de pessegueiros para esta finalidade. O objetivo deste trabalho foi avaliar os genótipos de pessegueiros ornamentais do programa de melhoramento da Embrapa Clima Temperado, quanto ao seu potencial para o uso no paisagismo. Foram observados o início, a plena e o final de floração, a tonalidade e o número de pétalas, o número de sépalas, a cor das folhas e quais genótipos produziram ou não frutos, no campo experimental da referida Instituição. Considerando os resultados obtidos e comparando-os com os observados para as cultivares já lançadas, 'Rosaflor' e 'Nectaflor', verificou-se que, nas progênies avaliadas, há genótipos que mantêm padrões ornamentais similares a essas cultivares e outros que as superam, tanto em número de pétalas, cor das folhas, e maior período de floração, sem produção de frutos. No geral, alguns genótipos destacam-se por complementarem a época de floração das cultivares anteriormente lançadas, ampliando o período disponível de flores, além da aparência igual ou superior às mesmas.

Termos para indexação: Prunus persica, Rosaceae, floração.

\section{EVALUATION OF ORNAMENTAL PEACH GENOTYPES FOR LANDSCAPING USE}

\begin{abstract}
Peach is generally planted because of the fruits; however there are genotypes interesting as ornamental plants and even some breeding lines going on for selecting new material specifically with this aim. The objective of this study was to evaluate the potential use for landscape of ornamental peach genotypes, originated from the Embrapa Clima Temperado peach breeding program. Beginning of blooming, full bloom and end of blooming were observed as well as color tonality and number of petals and sepals, leaf blade color and potential of fruit production. According to the obtained results and in comparison with the previous released cultivars, 'Rosaflor' and 'Nectaflor', it was concluded that several genotypes equal or surpass the standards in number of petals, leaf and petal color and longer blooming period, being several without fruit production. There are genotypes which could complement the blooming period of the released cultivars, extending the period of available flowers.
\end{abstract}

Index terms: Prunus persica, Rosaceae, blooming.

Originário da China, o pessegueiro é uma espécie frutífera de clima temperado. Pertencente à família rosácea, tem flores em geral, com cinco pétalas (RASEIRA;CENTELLAS-QUEZADAS, 2003). Há, entretanto genótipos que apresentam flores dobradas, isto é, um número de pétalas múltiplas de cinco. A beleza das delicadas flores de pessegueiro tem sido referida frequentemente, na literatura, desde os tempos antigos (HEDRICK, 1917). Há pessegueiros cultivados como plantas ornamentais e, inclusive, programas de melhoramento de pessegueiros para esta finalidade (YOSHIDA et. al., 2000).

A cor das pétalas também varia desde o branco até o vermelho, passando por várias tonalidades de rosa. As flores são completas e podem

ser solitárias ou agrupadas. Podem exibir a forma rosácea, com pétalas grandes, abertas e de coloração, geralmente, rosa-clara; ou campanulada, com pétalas muito pequenas e de coloração mais escura (SACHS; CAMPOS, 1998).

As folhas são oblongas, lanceoladas, com pecíolos curtos e distribuídas alternadamente. Medem, geralmente, de 10 a $50 \mathrm{~mm}$ de largura e de 140 a 180 $\mathrm{mm}$ de comprimento. As margens das folhas podem ser serrilhadas ou crenadas ou dentadas, sendo o limbo de coloração, em geral, verde durante o período de crescimento, havendo, entretanto, cultivares com folhas púrpuras ou variegadas. Plantas com cor púrpura nas folhas podem também ser interessantes para o paisagismo.

\footnotetext{
1(Trabalho 014-12). Recebido em: 03-01-2012. Aceito para publicação em: 06-03-2012.

${ }^{2}$ Eng. Agrônomo Msc. em Fruticultura de Clima Temperado, FAEM, UFPel, Pelotas-RS, telesca.tiagro@gmail.com

${ }^{3}$ Eng. Agrônomo, Dr. Pesquisadora Embrapa Clima Temperado, Bolsista CNPq, Pelotas-RS, bassols@cpact.embrapa.br

${ }^{4}$ Técnico Agrícola, Estagiário Embrapa Clima Temperado, Pelotas-RS, leonardogm92@gamil.com
} 
A Embrapa Clima Temperado tem, em seu programa de melhoramento de pessegueiro, uma linha para ornamentais, sendo que dessa, resultaram duas cultivares: a cultivar Nectaflor, lançada em 2002, e a cultivar Rosaflor, em 2004. As duas têm sido comercializadas por floriculturas locais (planta inteira em vaso).

Há ainda diversas seleções em avaliação. Algumas foram avaliadas por Romano et. al., 2009, quanto ao potencial para flor de corte.

O objetivo do trabalho foi avaliar os genótipos de pessegueiros ornamentais do programa de melhoramento da Embrapa Clima Temperado quanto ao seu potencial para o uso no paisagismo. Nesse estudo,foi avaliado um número maior de genótipos quanto ao uso em paisagismo, cujo potencial está ligado ao hábito de crescimento, à forma e à coloração das flores e à distinta coloração das folhas (YOSHIDA, etal., 2000, WANG et al., 2000 ;ROMANO et al., 2009).

Mas é também interessante ter plantas com floração escalonada, folhas de diferentes tonalidades e plantas estéreis para que não haja problemas com frutos podres ou atacados por mosca-das-frutas, necessitando tratamentos fitossanitários.

Assim, foram observados o início, a plena e o final de floração, a tonalidade e o número de pétalas, o número de sépalas, a cor das folhas e quais genótipos produzem frutos ou não os produzem. Fizeram parte destas observações, efetuadas no campo experimental da Embrapa Clima Temperado: as duas cultivares já lançadas, Nectaflor e Rosaflor; oito "seedlings" oriundos do cruzamento de 'Cerrito' $x$ 'Pink cascade' (C-99-34); cinco "seedlings" oriundos do cruzamento 'Sunred' x 'Pink cascade' (C-99-88); dez "seedlings" originários por polinização livre (F2) do cruzamento de 'Necta 383' x 'Chiripá' (C-2003-220) e quarenta e cinco "seedlings" oriundos do cruzamento "Exo6' x 'Nectaflor' (C-2003-35). Nas duas cultivares, Rosaflor e Nectaflor, e nos genótipos das progêneas C-99-34 e C-99-88, as observações foram efetuadas em plantas enxertadas sobre porta-enxertos oriundos de sementes e plantadas no local definitivo, em 2005, sendo avaliadas três plantas por genótipo. Já os genótipos das progênies C-2003-35 e C-2003-220 foram observados nos "seedlings" originais, sendo uma planta por genótipo, que havia sido transplantado, em 2004.

O início da floração foi estimado quando a planta se encontrava com cerca de $10 \%$ de flores abertas, a plena floração quando mais de $50 \%$ das flores estavam abertas e o final da floração quando, praticamente, não havia flores por abrir, e a maioria já estava perdendo as pétalas.

Para determinar o número de pétalas e sépalas, foram contadas três flores de cada genótipo. Se os números fossem variáveis (o que geralmente não acontecia) então, eram contadas as pétalas em um número maior de flores.
Para a cor das pétalas, foram conferidos graus, sendo G1 - Rosa-clara, G2 - Rosa-média, G3 - Rosa escura e G4 - Pink (entre o rosa escuro e o vermelho). Posteriormente, foi verificado quais progênies frutificaram ou não.

Os dados relativos ao período de floração encontram-se na Figura 1. De modo geral, as plantas oriundas do cruzamento 220 ('Necta 383'x 'Chiripá' $\mathrm{x} \mathrm{Pl}$ ), tiveram floração, mais precoce.

Os seedlings C-2003-35-18, C-2003-35-24; C-2003-35-31 e C-2003-35-45 tiveram os mais curtos períodos de floração enquanto C-99-88.25 apresentou o período mais longo.

Como também havia sido constatado por Romano et al., 2009, a plena floração da cv. Rosaflor ocorreu alguns dias (4 - 5) mais tarde que da cv. Nectaflor. Os genótipos comuns ao trabalho de Romano et al., 2009, tiveram floração mais tardia no ano de 2010 do que nas observações efetuadas por aqueles autores.

Apenas cinco genótipos apresentam folhas púrpuras (exceto ao final do ciclo, quando se tornam verdes), mas deles apenas C-99-34-25 e C-99-12 têm flores dobradas, com até 30 pétalas por flor (Tabela 2).

$\mathrm{O}$ número de pétalas variou de cinco nos genótipos C-99-34-5, C-99-34-6 e C-99-88-1 a trinta nos genótipos C-99-34-15, C-99-88-25 e C-2003220-6 (Tabela 1). É importante salientar que todos os "seedlings" do cruzamento C-2003-35 têm pétalas dobradas, e dez sépalas por flor. Nesta progênie, destaca-se o genótipo C-2003-35-44, que possui uma cor mais intensa, mais escura e não produz frutos (Tabela 1).

Nos 70 genótipos observados, o número de sépalas foi de 5 ou de 10 , sendo que os genótipos com o número de sépalas duplas mantêm aspecto ornamental mesmo depois de as pétalas caírem (Tabela 1).

Considerando os resultados obtidos e comparando-os com os observados para os padrões 'Rosaflor' e 'Nectaflor', há, dentro das progênies avaliadas, genótipos que mantêm padrões ornamentais similares a essas cultivares e outros que as superam, tanto em número de pétalas, cor das folhas, quanto ao maior período de floração, sem produção de frutos (Figura 1 e Tabela 1).

No geral, destacam-se os genótipos C-2003220-6, C-2003-220-9, C-2003-220-12 e C-2003-22018 que, pela época de floração, complementam as cultivares já lançadas, ampliando o período de flores disponíveis além de ter aparência igual ou superior às mesmas. Merece destaque também o C.2003.35.44, pela cor mais escura das pétalas, e a C-99-34-25, pela coloração das folhas, além do número de pétalas.

Este trabalho é uma pequena contribuição a uma área muito deficiente no Brasil, já que, como salientam Castan,(2005) e PEREIRA et al. (2006), há poucos trabalhos publicados referindo- à produção de flores e plantas ornamentais.. 


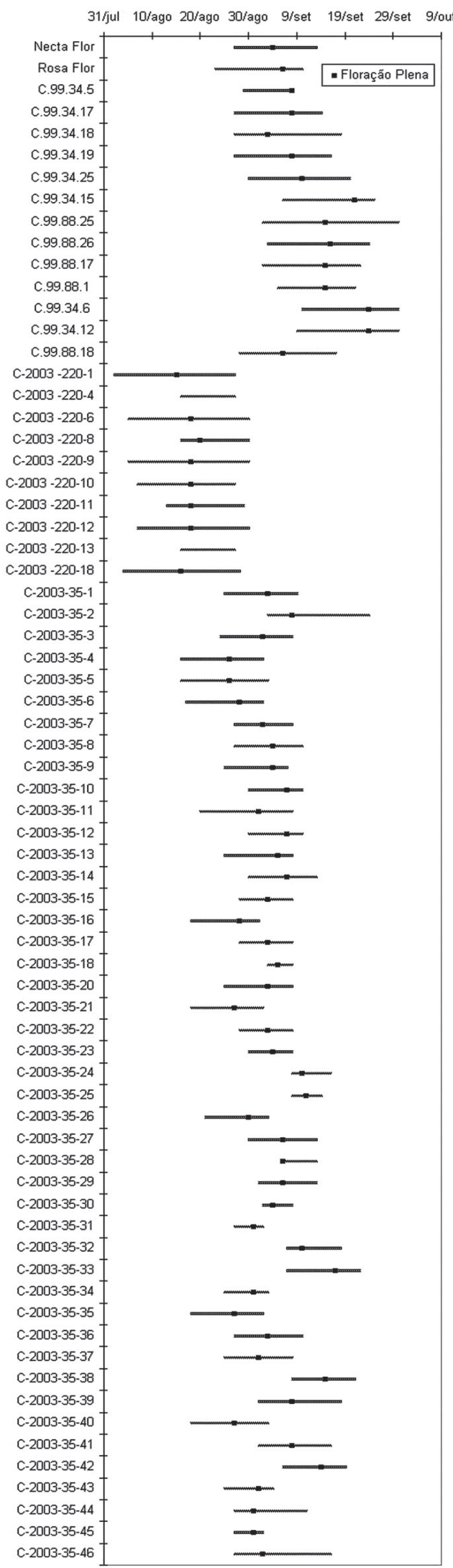

FIGURA 1 - Dados de época de Floração de diversos genótipos ornamentais de pessegueiro, no ano de 2010, Embrapa Clima Temperado, 2011. 

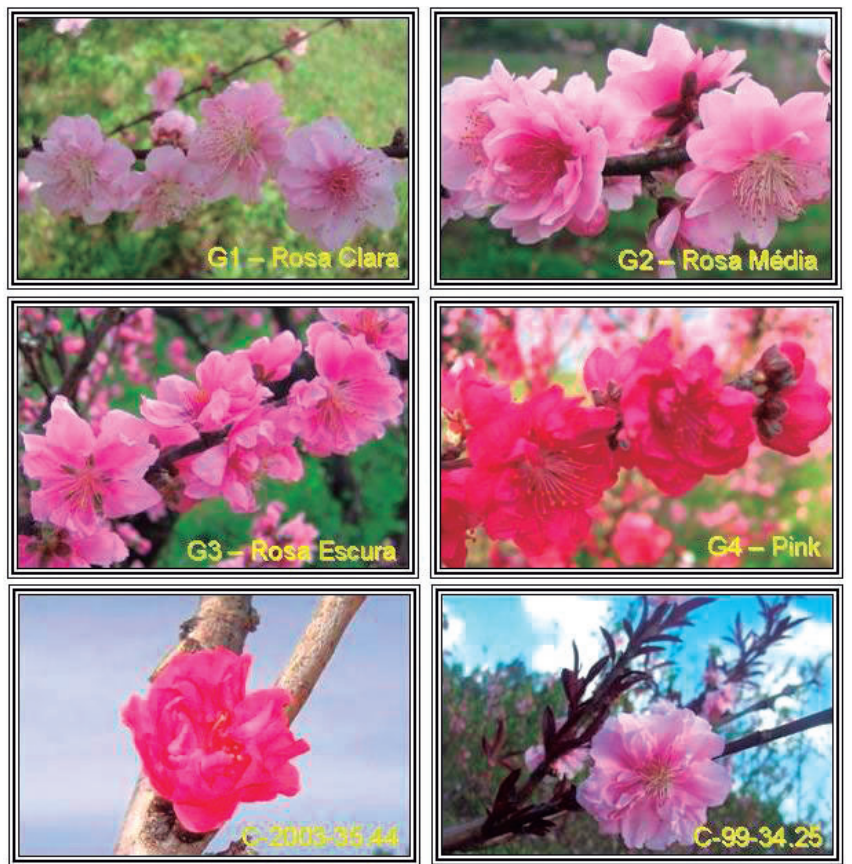

FIGURA 2- Graus conferidos à cor das pétalas, sendo: G1 - rosa clara; G2 - rosa média; G3 - rosa escura, e G4 - pink (entre o rosa escuro e o vermelho) e C-2003-35-44, mostrando a cor das pétalas mais escura, e o C-99-34.25 com destaque para as folhas púrpuras e no número de pétalas. Embrapa Clima Temperado, 2011.

TABELA 1- Dados obtidos em 2010, para número médio de pétalas e número médio de sépalas por flor; coloração das pétalas e produção de frutos nas cvs. Nectaflor e Rosaflor e genótipos das progênies C-99.34; C-99-88, C-2003 -220 e C-2003-35.Embrapa Clima Temperado, 2011.

\begin{tabular}{lccll}
\hline \multicolumn{1}{c}{ Cultivar ou Seleção } & $\mathbf{N}^{\mathbf{0}}$ de Pétalas & $\mathbf{N}^{\circ}$ de Sépalas & \multicolumn{1}{c}{ Cor } & Produção \\
\hline Necta Flor & 19 a 20 & 10 & Pink & Sim $(\mathrm{P})$ \\
Rosa Flor & 19 a 21 & 10 & Rosa média & Sim \\
C.99.34.5 FP & simples & & Rosa clara & Sim \\
C.99.34.17 & 25 & 10 & Rosa média & Sim (P) \\
C.99.34.18 & 20 a 22 & 5 & Rosa clara & Sim (P) \\
C.99.34.19 & 25 a 28 & 5 & Rosa média & Sim \\
C.99.34.25 FP & 28 a 29 & 5 & Rosa média & Sim (P) \\
C.99.34.15 & 25 a 30 & 5 & Rosa média & Não \\
C.99.34.6 FP & simples & & Rosa clara & \\
C.99.34.12 FP & 27 a 29 & 5 & Rosa média & Sim (P) \\
C.99.88.25 & 25 a 30 & 5 & Rosa média & Sim \\
C.99.88.26 & 25 & 5 & Rosa média & Sim \\
C.99.88.17 & 28 a 29 & 5 & Rosa média & Sim \\
C.99.88.1 FP & simples & & Rosa clara & \\
C.99.88.18 & 20 a 25 & 5 & Rosa clara & \\
C-2003 $-220-3$ & simples & & & Sim \\
C-2003 -220-4 & simples & & & Sim \\
C-2003 -220-6 & 25 a 30 & 10 & Rosa escura & Sim \\
C-2003 -220-8 & simples & & Rosa média & Sim \\
C-2003 -220-9 & 25 & 10 & Rosa média & Sim \\
C-2003 -220-10 & 7 a 12 & 5 & Rosa média & Sim \\
C-2003 -220-11 & 7 a 10 & 5 & Rosa média & Sim \\
\hline
\end{tabular}


continuação...

\begin{tabular}{|c|c|c|c|c|}
\hline C-2003 -220-12 & 20 a 25 & 10 & Rosa escura & Sim \\
\hline C-2003 -220-13 & simples & & & Sim \\
\hline C-2003 -220-18 & 14 a 16 & 10 & Rosa escura & Sim \\
\hline C-2003-35.1 & 16 a 19 & 10 & Pink & Sim \\
\hline C-2003-35.2 & 18 a 23 & 10 & Pink & Não \\
\hline C-2003-35.3 & 15 a 19 & 10 & & Sim \\
\hline C-2003-35.4 & 15 a 18 & 10 & Rosa média & Sim \\
\hline C-2003-35.5 & 15 a 18 & 10 & Rosa média & Sim \\
\hline C-2003-35.6 & 15 a 19 & 10 & Rosa média & Sim \\
\hline C-2003-35.7 & 19 a 22 & 10 & Pink & Sim \\
\hline C-2003-35.8 & 15 a 18 & 10 & Rosa média & Sim \\
\hline C-2003-35.9 & 15 a 17 & 10 & Rosa média & Sim \\
\hline C-2003-35.10 & 15 a 20 & 10 & Pink & Não \\
\hline C-2003-35.11 & 15 a 20 & 10 & Rosa média & Sim \\
\hline C-2003-35.12 & 15 a 20 & 10 & Pink & Não \\
\hline C-2003-35.13 & 10 a 16 & 10 & Rosa média & Sim \\
\hline C-2003-35.14 & 13 a 16 & 10 & Pink & Não \\
\hline C-2003-35.15 & 16 a 19 & 10 & pink & $\operatorname{Sim}(\mathrm{MP})$ \\
\hline C-2003-35.16 & 16 a 19 & 10 & Rosa média & Sim \\
\hline C-2003-35.17 & 15 a 20 & 10 & Pink & $\operatorname{Sim}(\mathrm{MP})$ \\
\hline C-2003-35.18 & 16 a 18 & 10 & Rosa média & $\operatorname{Sim}(\mathrm{MP})$ \\
\hline C-2003-35.20 & 15 a 16 & 10 & Rosa escura & $\operatorname{Sim}(\mathrm{MP})$ \\
\hline C-2003-35.21 & 12 a 16 & 10 & Rosa média & Sim \\
\hline C-2003-35.22 & 16 a 21 & 10 & Rosa clara & Sim (MP) \\
\hline C-2003-35.23 & 10 a 16 & 10 & Pink & $\operatorname{Sim}(\mathrm{MP})$ \\
\hline C-2003-35.24 & 12 a 16 & 10 & Rosa média & Sim \\
\hline C-2003-35.25 & 8 a 15 & 10 & Rosa clara & Não \\
\hline C-2003-35.26 & 12 a 16 & 10 & Rosa clara & Sim \\
\hline C-2003-35.27 & 12 a 15 & 10 & Rosa escura & Sim \\
\hline C-2003-35.28 & 22 a 24 & 10 & Rosa média & Não \\
\hline C-2003-35.29 & 10 a 12 & 10 & Rosa média & Sim \\
\hline C-2003-35.30 & 10 a 16 & 10 & Rosa média & Sim \\
\hline C-2003-35.31 & 12 a 14 & 10 & Rosa média & Sim \\
\hline C-2003-35.32 & 16 a 19 & 10 & Pink & Sim \\
\hline C-2003-35.33 & 15 a 17 & 10 & Pink & $\operatorname{Sim}(\mathrm{MP})$ \\
\hline C-2003-35.34 & 12 a 13 & 10 & Rosa média & Sim \\
\hline C-2003-35.35 & 16 a 20 & 10 & Rosa média & Sim \\
\hline C-2003-35.36 & 17 a 19 & 10 & Rosa média & Sim \\
\hline C-2003-35.37 & 16 a 20 & 10 & Rosa claro & Sim \\
\hline C-2003-35.38 & & 10 & Pink & Não \\
\hline C-2003-35.39 & 13 a 15 & 10 & Pink & $\operatorname{Sim}(\mathrm{MP})$ \\
\hline C-2003-35.40 & 16 a 25 & 10 & Rosa clara & Sim \\
\hline C-2003-35.41 & 15 a 19 & 10 & Rosa clara & Sim \\
\hline C-2003-35.42 & 14 a 16 & 10 & Pink & $\operatorname{Sim}(\mathrm{MP})$ \\
\hline C-2003-35.43 & 15 a 18 & 10 & Rosa média & Sim \\
\hline C-2003-35.44 & 10 a 18 & 10 & Pink + & Não \\
\hline C-2003-35.45 & 16 a 18 & 10 & Rosa média & Sim (MP) \\
\hline C-2003-35.46 & 13 a 16 & 10 & Rosa média & $\mathrm{Sim}$ \\
\hline
\end{tabular}




\section{REFERÊNCIAS}

HEDRICK, U.P. The peaches of New York. In: ANNUAL REPORT, 24., 1917, Albany. Proceedings... Albany: J.B. Lyon Company, 1917, v.2, 541p.

PEREIRA, J. B.; CAVALLET, V. J.; FONTE, N. N.; BORSATTO, R.; OTTMANN, A. M. M.; LOURENÇATO, L. F. Análise da preocupação social e ambiental das pesquisas realizadas pelo Setor de Ciências Agrárias da UFPR.. In: EVENTO DE INICIAÇÃO CIENTÍFICADA UFPR, 14., 2006, Curitiba. Anais... Curitiba: UFPR, 2006.

RASEIRA, A.; CENTELLAS-QUEZADA, A. Classificação botânica, origem e evolução. In: EMBRAPA. Pêssego produção. Brasília: Embrapa/ Informação Tecnológica, 2003. 162p.
ROMANO, C. M.; RASEIRA, M. C. do B.; NEITZKE, R.S.; STUMPF, E. R. T.; BARBIERI, R.L. Caracterização de árvores de pessegueiros ornamentai. Magistra, Cruz das Almas, v. 21, n. 4, p.268-276, 2009

SACHS, S.; CAMPOS, A.D. O Pessegueiro In: MEDEIROS , C. A. B. ; RASEIRA , M. do C. A cultura do pessegueiro. Brasília: Embrapa- CPACT, 1998. p. 13-19.

WANG, L.; ZHU, G.; ZUO, Q. Evaluation of the ornamental value of peach flowers. Journal of Fruit Science, Philadelphia, v.17, p.8-14, 2000. Suplement

YOSHIDA, M.; YAMANE, K.; FUJISHIGE, N. Ornamental peach cultivars in Japan. In: PRUNUS BREEDERS MEETING. Pelotas: Embrapa Clima Temperado, 2000. p 33-34. 